\title{
The development of hybrid electric vehicle control strategy based on GT-SUITE and Simulink
}

\author{
Chunying Chen \\ Department of Mechanical and Electronic, Handan Polytechnic College, Handan, 056000, China \\ email: ccy6825@126.com
}

Keywords: Hybrid; Simulation Analysis; GT-SUITE; Simulink

\begin{abstract}
Hybrid electric vehicle is the car which increase a electric drive system on the traditional internal combustion engine vehicle. The coordination work based on the drive motor and the internal combustion engine can reduce fuel consumption and $\mathrm{CO} 2$ emission. Considering on the special characteristics and power system structure, we need a integrated control system to optimise the energy distribution. In this paper, based on the GT-SUITE software in the stage of system concept design validate components parameters meet the performance indicators, based on the Simulink software in the control system development stage establish a vehicle energy management strategy, then synthetise the two systems to optimise the fuel economy of the whole vehicle.
\end{abstract}

\section{Introduction}

In order to make full use of the advantages of hybrid electric vehicle on energy saving and high efficiency, there must be a complete set of control strategy to ensure the best way of coordination between the motor drive system and engine drive system. The formulation of hybrid control strategy, using vehicle performance index and power system component as input parameters, through the optimization of the parts working characteristics at different time, achieve the intent of the driver's operation. In this paper, in the vehicle design conceptual stage, use the GT-SUITE software to analyse the control strategy for the vehicle design, optimise the torque control of the power system, provide the upper strategy and functional definition for ECU development.

\section{Research object}

This paper studies the plug-in hybrid electric vehicle powertrain system structure as shown in Figure 1, which is based on the existing car platform, and make some design and development of adaptability. The original engine power transmission system is replaced by the new development of hybrid drive system. The hybrid system compounds both advantages of series and parallel hybrid power system, according to the different needs of vehicle driving, to make each power source in the best condition. The driving motor has two working modes which can be used as a motor or a generator. The motor is used as a generator in the braking energy recovery condition in order to

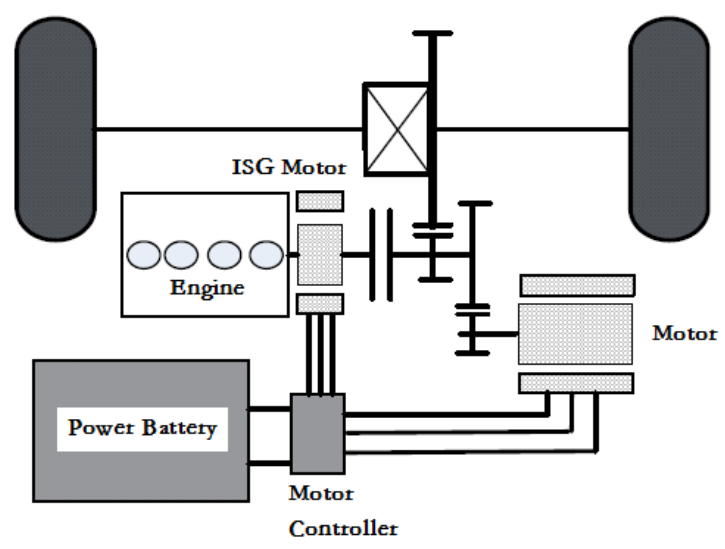

Fig.1 The vehicle structure system 
charge the battery recycling braking energy, In the drive mode it is used as a motor, suppling the battery electricity to drive the motor and the wheel. ISG motor can also be used as a motor or a generator, when working at acceleration process or engine start conditions, the ISG motor works as a motor improve vehicle power or start the engine. In the low SOC condition, ISG motor works as generator to charge the battery maintaining the state of SOC.

\section{Working mode}

The operation condition of hybrid car mainly includes: starting, accelerating, climbing, braking, idling etc.. Due to the differences of operation characteristics between the engine and the motor, they have different high efficiency areas, so in order to make full use of both advantages of the hybrid system, to make the engine and the motor working more efficiently, the car should have a variety working modes to meet different conditions, so as to improve the performance of the whole vehicle. According to the different working requirement and energy allocation scheme, the HEV mode can be divided into six basic operating modes: motor drive mode, engine driving mode, engine and motor driving mode, engine driving and charging mode, extended range mode, braking energy recovery mode and idle / parking mode.

(a) Motor driving mode. When the vehicle is started or in low speed light load condition, because of the engine performance characteristic, the engine fuel efficiency is low and the emission is poor at that time. Therefore, if the battery power is sufficient, then turn off the engine, the battery electric motor supplied the power which can drive the vehicle starting or running. If the battery power is low, to the protect the battery service life the control system should be switched to extended range mode.

(b) Engine driving mode. When the vehicle runs at a high speed medium load condition, by the automobile theory, the driving force required to overcome the vehicle running resistance is small, then start the engine and provide the vehicle driving force by the engine. In order to reduce fuel consumption and achieve emission targets, it should make sure that the engine worked in the high efficiency region as much as possible.

(c) Engine and the motor driving mode. When the vehicle is accelerating or climbing with high load, usually the engine does not provide sufficient driving force which vehicle required, then make the engine worked in the high efficiency area, the lack of driving force is provided by the motor or ISG motor, while the engine is combined with motor for driving vehicle. If the state of the battery power is low, then converse to the engine driving mode.

(d) Engine driving and charging mode. When the vehicle is in normal running and low load, if the battery group's remaining power is low, then start the engine, the engine need provide the vehicle driving power which is required for the output power, but also to provide additional power to charge the battery pack through the generator.

(e) Extended range mode. When the vehicle speed is low, the driving motor drives the vehicle, engine and ISG motor control strategies worked in thermostat type, the engine drive the ISG motor to work as generator for the battery charging.

(f) Braking energy recovery mode. When the vehicle is in braking condition, turn off the engine, motor maximumly recovery the braking energy, the remaining part of the braking energy is consumpted by the mechanical brake system.

(g) The idle / parking mode. When the vehicle is idling, shut down the engine and motor, when the remaining battery group's power is low, then start the engine, control the engine working in the high efficiency area, make the engine driving the generator to charge the batteries.

\section{Modeling}

According the construction of the whole power system to build the model in the GT-SUITE software environment. The model include: engine module, drive motor module, ISG motor module, battery module, car body module and transmission module. Then built the mechanical and electrical signaling connection which is the final step of vehicle structure modeling. In order to make the 
power system components running in the requirements of the driver, the corresponding control module is needed. According to the needs of the system, the control module comprised vehicle control module (HCU), engine control module (ECU), motor control module (MCU) and battery control module (BMS) etc.. With the vehicle model and control model in GT-SUITE as shown in figure 2.

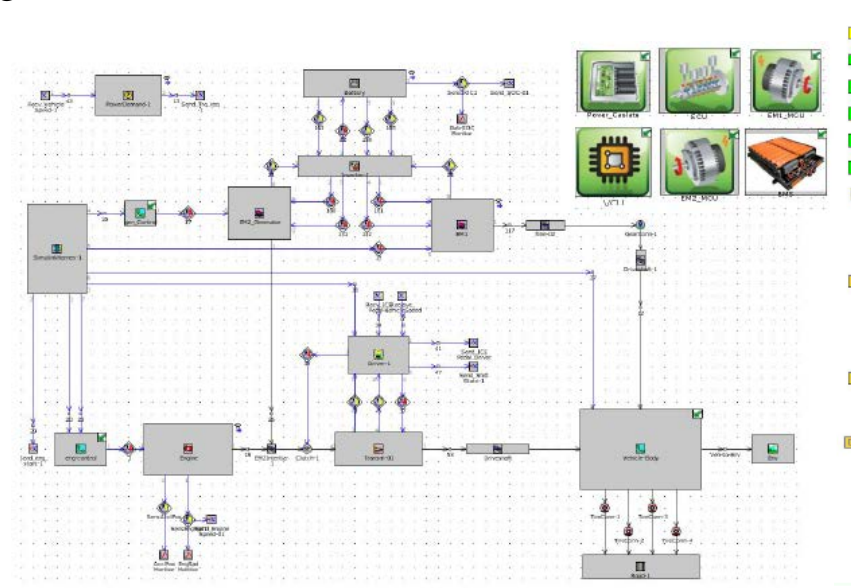

Fig.2 GT-SUITE Model

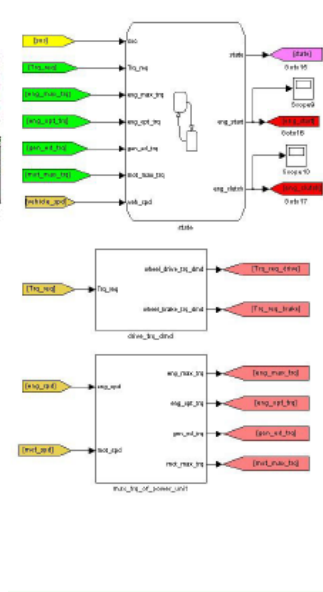

Fig.3 Simulink Model

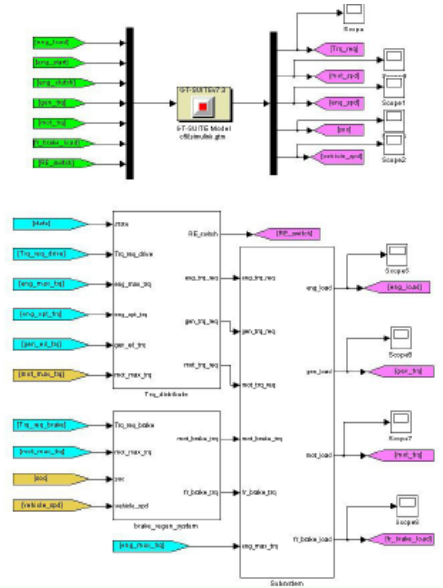
According to the working mode mentioned above, we can build a control strategy model, in the
imulink software environment include: demand torque calculation module, work mode judging module, energy distribution calculation module, braking energy recovery control module and signal input and output module etc.. The simulink control strategy model is as shown in figure 3.

\section{The optimization process}

In the control strategy formulation process, use the control strategy optimization process as the guidance. Firstly, the simulation model is established in the GT-SUITE software, and then with the conditions and strategies setted already to simulate the fact and verify the model. Evaluate and analyse the simulation results and parts' working state. After the optimization process with comparative analysis, ultimately determine the control strategy. As shown in figure 4.

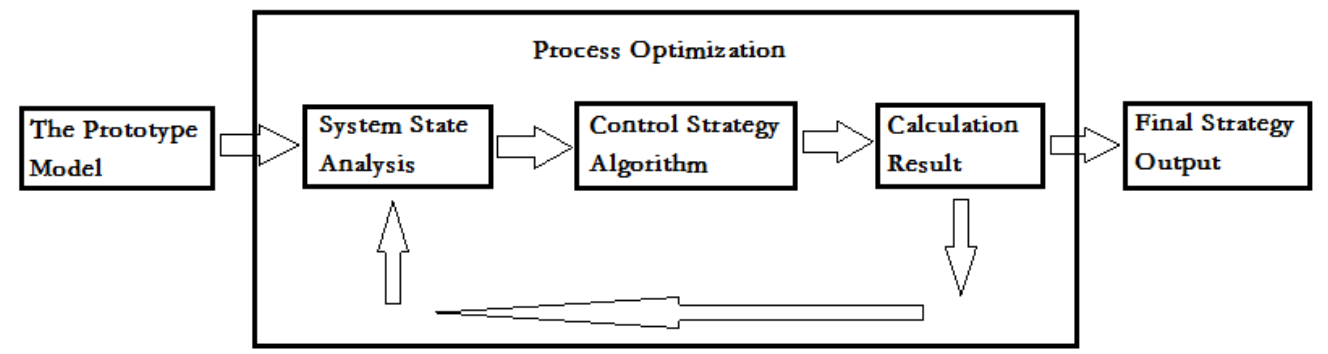

Fig.4 The optimization process

\section{Result}

Select the NEDC cycle as the road cycle, analysis the simulation result of vehicle fuel economy. Optimise the control strategy based on the analysis of simulation results, to ensure the engine work points at goal setting position. The simulation results of vehicle driving fuel consumption is as shown in figure 5 . And the contrast between simulation and experiment is as shown in table 1.

Tab.1 Contrast between simulation and experiment

\begin{tabular}{|c|c|c|}
\hline & Test Result & Simulation Result \\
\hline $\begin{array}{c}\text { Fuel consumption } \\
\text { L/100km }\end{array}$ & 4.79 & 4.81 \\
\hline Pure electric driving mileage $\mathrm{km}$ & 51 & 52 \\
\hline
\end{tabular}




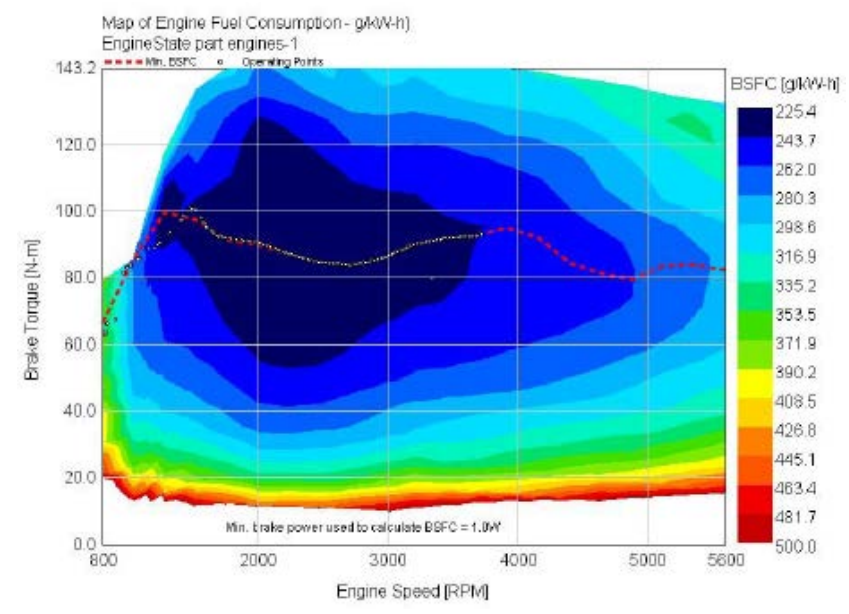

Fig.5. The simulation curve of fuel consumption

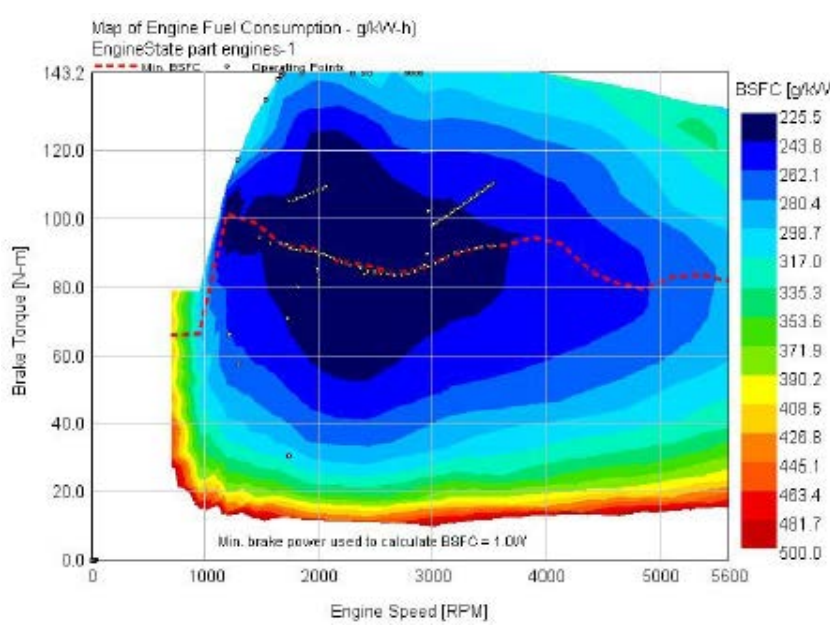

Fig.6.Fuel consumption with control strategy optimization

The simulation contrast result above means that the model can coincide the true situation very well. Use the model as basis of the electric vehicle control strategy optimization, in order to reduce the fuel consumption of 100 kilometers. The result is shown in figure 6 .

Fuel consumption comparison before and after optimization are shown in table 2.

Tab.2 Optimization result

\begin{tabular}{|c|c|c|}
\hline & Before & Final \\
\hline $\begin{array}{c}\text { Fuel consumption } \\
\text { L/100km }\end{array}$ & 4.81 & 4.48 \\
\hline
\end{tabular}

The simulation result shows that the control strategy can effectively allocate demand torque engine based on optimal working line, and control the output torque of engine and motor coordination reasonably. Achieve better fuel economy when meeting the dynamics performance of the vehicle as prerequisite

\section{Conclusion}

According to the new development of hybrid power system and the characteristics of power source, we established the vehicle operating mode and energy distribution algorithm, using GT-suite software and Simulink software respectively built the vehicle simulation model and control strategy model, then made a compound simulation. We analysed the simulation results and the work state of the power sources, in oder to optimise the control strategy algorithm. So each power source can work at the best condition, reaching the purpose of reducing fuel consumption and emissions. At the same time, in the control system development stage, we can evaluate the control schemes efficiently and finally reduce the development cycles.

\section{References}

[1] Chen Qingquan, Sun Fengchun. Hybrid electric vehicle foundation. Beijing Institute of Technology Press. 2001

[2] Wang Haibin, Yu Shui. The control strategy for parallel hybrid electric vehicle and engine optimization. Small internal combustion engine and motorcycle. 2008

[3] Mehrdad Ehsani , Yimin Gao, Ali Emadi . Modern Electric , Hybrid Electric, and Fuel Cell Vehicles Fundamentals, Theory , and Design Second Edition. CRC Press, 2010

[4] Liu Xiaokang, Wang Bin, Yu Xiangdong, Wu Jieyu. A parallel hybrid electric vehicle battery parameter optimization. Automotive engineering. 2013,29 (7).

[5] Zhou Nenghui, Zhao Chunming, Xin Minghua, Li Lei, Xia Chaoying. Study on the control strategy of the plug-in hybrid electric vehicle. Automotive engineering. 2013,35 (2). 